\title{
Genetics of life history in Daphnia magna. II. Phenotypic plasticity
}

\author{
DIETER EBERT*, LEV YAMPOLSKY† \& ARIE J. VAN NOORDWIJK $\ddagger$ \\ Institut für Zoologie, Universität Basel, Rheinsprung 9, CH-4051 Basel, Germany
}

\begin{abstract}
The phenotypic plasticities of life-history traits in 46 clones of the planktonic crustacean Daphnia magna were measured across two feeding conditions. Plasticity estimates for clutch sizes and offspring lengths of the first six clutches and for lengths of eight successive adult instars allowed us to compare plasticity between and within these three trait groups. Data were standardized (mean $=0$, variance $=1$ ) in each environment before analysis. The broad-sense heritability of plasticity of adult length increased from about 0 in the adolescent instar to 60 per cent in the 7 th adult instar, while narrow-sense heritability was low for all instars. For clutch sizes, narrow- and broad-sense heritabilities were around 25 per cent. For offspring length, they were mostly close to zero. A comparison of three methods of quantifying plasticity showed that the heritabilities of trait differences across environments and the heritabilities based on genotype by environment interaction components were consistent with each other, but the later is always smaller. Crossenvironment genetic correlations gave qualitatively different results.
\end{abstract}

Keywords: Cladocera, Daphnia, genetic correlation, heritability, plasticity, reaction norm.

\section{Introduction}

Observing the phenotypes that a single genotype expresses over a range of different environments is the easiest way to visualize the concept of reaction norm. Woltereck (1909) used helmet length under different feeding conditions in a clone of Daphnia cucullata to illustrate the term 'Reaktionsnorm', the norm of reaction or phenotypic plasticity, when he used it for the first time. For evolutionary biologists, this concept is helpful in two contexts. First, it aids understanding of the ecological consequences of expressing different phenotypes in different environments, e.g. predatorinduced defense (e.g. Appleton \& Palmer, 1988; Dodson, 1989) and secondly, genetic variation in reaction norms has important consequences for evolution, especially in heterogeneous or unstable environments (e.g. Stearns, 1989; van Noordwijk, 1989).

*Correspondence and present address: University of Oxford, Department of Zoology; South Parks Road, Oxford, OX1 3PS, UK.

†Present address: N. I. Vavilov Institute of General Genetics, Academy of Science of Russia, Gubkin str., 3 GSP-1, V-333; Moscow, Russia.

$\ddagger$ Present address: Instituut voor Oecologisch Onderzoek, Postbus 40; NL-6666 ZG Heteren, Netherlands.
Although the genetics of phenotypic plasticity has been studied for many years (e.g. Becker, 1964; Robertson, 1960; Gupta \& Lewontin, 1982; Via, 1984; Scheiner \& Goodnight, 1984; Gebhardt \& Stearns, 1988) the interpretation of the statistical results still causes problems (e.g. Scheiner \& Lyman, 1989; Scheiner et al., 1991). There is a need for studies comparing different methods to quantify genetic variation of reaction norm.

Clonal organisms allow one to raise individuals of the same genotype under different environmental conditions. In the present study we used clones of Daphnia magna, which were kept in two feeding conditions, to quantify genetic variation in plasticity. Life-history traits were analysed separately in successive adult instars, which allowed us to identify trends in plasticity during development and to judge the reliability of estimates.

\section{Materials and methods}

We conducted life-history experiments at two food levels with 23 mother and 23 sexually produced daughter clones of Daphnia magna. Experiments with three replicates per clone were performed in high and in low food concentration. The details of the experi- 
mental work are described in the accompanying paper (Ebert et al. 1993a; see previous paper).

\section{Data analysis}

Phenotypic plasticity can be analysed in various ways (e.g. Scheiner \& Lyman, 1989). We used the trait difference between the two environments, but crossenvironment genetic correlations and genotype by environment interaction components (from 2-way ANOVA) were used for comparisons.

\section{Heritability estimates of cross-environment differences}

The differences between trait values expressed in two environments measure the plasticity of this trait and can be analysed like life-history traits within environments (Scheiner et al., 1991). Variance component analyses with these differences were done using the maximum-likelihood method (PROC VARCOMP; SAS, 1985). Following Scheiner et al. (1991) we call this estimate the 'heritability of plasticity', $H_{\mathrm{pl}}^{2}$. It is basically the same approach taken by Gavrilets (1986) and de Jong (1990). They describe a reaction norm as the slope of a linear regression of the trait values on the environment.

Since it is impossible to obtain a measure of a trait from the same individual simultaneously in two environments, we randomly assigned single daphniids raised in high food conditions to single daphniids of the same clone raised in low food conditions. Then we subtracted the low food trait value from the high food value. By this method we obtained three measures of trait difference per clone. These differences were used in the analysis. We used parent-offspring regression and variance component analysis to estimate variance components due to genetic and additive genetic effects (see accompanying paper for details of this method; (Ebert et al., 1993)). We calculated broad- and narrow-sense heritability (Falconer, 1981) for all 21 traits.

Using the differences between traits from two environments introduces a scaling problem. If variances in both environments are markedly different, then the difference is dominated by the trait value in the environment with the larger variance. In our data, the total variance of some traits differed markedly between environments, e.g. clutch sizes and adult lengths had much larger variances in high than in low food. For this reason we standardized each trait by subtracting the mean from each value and dividing it by the standard deviation $($ mean $=0$, variance $=1)$. The effect of the transformation is to emphasize, not the absolute value of a trait in an environment, but its relative value. We repeated the analysis of plasticity with the standardized data.

\section{Comparison of methods}

For comparison of methods we used only the 46 clones, ignoring the family structure of the dataset. This was done because most additive variance estimates were not significant and also because it kept the comparisons more transparent. Using all clones resulted in somewhat different, but more accurate (doubled sample size), estimates compared with those used for the analysis of broad-sense heritability of plasticity (see above and Ebert et al., 1993), because for the latter only mother clones were included, which was necessary for comparison with mother-daughter regression estimates, which estimate additive variance only for mothers.

Using the standardized data we calculated: (i) the clonal variance component of the cross-environment difference, $H_{\mathrm{p} 1}^{2}$, as well as the within environment heritabilities (1-way ANOVAs with clone as main effect, maximum-likelihood method; SAS, PROC VARCOMP); (ii) the variance component of the genotype by environment interactions term $V_{\mathrm{G} \times \mathrm{E}}$, from two-way mixed model ANOVAs with food level as fixed effect and clones as random effect (maximumlikelihood method; SAS, PROC VARCOMP); (iii) the cross-environment correlations (CEC) of clonal means, using the Pearson correlation coefficient; and (iv) the cross-environment correlations of clonal means, using the Spearman correlation coefficient. All variance components are given as percentages of the total variance.

\section{Statistical interdependence of quantification methods}

The mean squares (MS), and the corresponding variances obtained from a 1-way ANOVA with trait difference across two environments as main effect and the MS of a two-way ANOVA with clone and environment as main effects (both ANOVAs as described in the previous section) relate in the following way to each other:

$$
\begin{gathered}
\mathrm{MS}_{\mathrm{D}}=\sigma_{\mathrm{e}}^{2}+n \sigma_{\mathrm{D}}^{2}=2 \mathrm{MS}_{\mathrm{G} \times \mathrm{E}}=2\left(\sigma_{\text {error }}^{2}+n \sigma_{\mathrm{G} \times \mathrm{E}}^{2}\right) \\
\mathrm{MS}_{\mathrm{e}}=\sigma_{\mathrm{e}}^{2}=2 \mathrm{MS}_{\text {error }}=2 \sigma_{\text {error }}^{2},
\end{gathered}
$$

where $\sigma_{\mathrm{e}}^{2}$ and $\sigma_{\mathrm{D}}^{2}$ are the variances of the error and the trait difference from the one-way ANOVA (note the use of one letter subscripts for one-way ANOVAs), $n$ is the number of clonal replicates within each environment ( $n=3$ in our design) and the terms with 
the subscripts error and $G \times E$ refer to the mean squares (MS) and variances $\left(\sigma^{2}\right)$ of the error and the interaction term from the two-way ANOVA, respectively. The variance of the clone effect from the two-way ANOVA has no corresponding term in the one-way ANOVA, since the mean clone effect disappears when differences across environments are calculated. The variance of the clone therefore represents the genetic covariance $\mathrm{COV}$, across the two environments.

$\sigma_{\text {clone }}^{2}=\mathrm{COV}$

(equation 1)

From the mean square comparison above it follows that

$\sigma_{\mathrm{D}}^{2}=2 \sigma_{\mathrm{G} \times \mathrm{E}}^{2}$

(equation 2).

The genetic variance of the difference $D$, between the high food, and the low food environment is

$\sigma_{\mathrm{D}}^{2}=\sigma_{\text {High }}^{2}+\sigma_{\text {Low }}^{2}-2 \mathrm{COV}$

(equation 3)

(Sokal \& Rohlf, 1981), where $\sigma_{\text {High }}^{2}$ and $\sigma_{\text {Low }}^{2}$ are the genetic variances in the two environments. From equations 1,2 and 3 it follow that

$\sigma_{\mathrm{D}}^{2} / 2+\mathrm{COV}=\sigma_{\mathrm{G} \times \mathrm{E}}^{2}+\sigma_{\text {clone }}^{2}=\left(\sigma_{\text {High }}^{2}+\sigma_{\text {Low }}^{2}\right) / 2$.

The relation of the error terms are similar, but since errors across environments are assumed to have no covariance the equation for the error terms simplifies to

$\sigma_{\mathrm{e}}^{2} / 2=\sigma_{\text {error }}^{2}=\left(\sigma_{\text {Herror }}^{2}+\sigma_{\text {Lerror }}^{2}\right) / 2$,

where $\sigma_{\text {Herror }}^{2}$ and $\sigma_{\text {Lerror }}^{2}$ are the error terms within the high and the low food environment.

The heritability of plasticity is obtained from

$H_{\mathrm{p} 1}^{2}=\frac{\sigma_{\mathrm{D}}^{2}}{\sigma_{\mathrm{D}}^{2}+\sigma_{\mathrm{c}}^{2}}$,

which is equal to

$\frac{2 \sigma_{\mathrm{GXE}}^{2}}{2 \sigma_{\mathrm{G} \times \mathrm{E}}^{2}+2 \sigma_{\text {error }}^{2}}$.

Cancelling the 2 in the term on the right-hand side we obtain a term which is similar to the formula for the heritability based on the $\mathrm{G} \times \mathrm{E}$ component

$\mathrm{V}_{\mathrm{G} \times \mathrm{E}}=\frac{\sigma_{\mathrm{G} \times \mathrm{E}}^{2}}{\sigma_{\text {clone }}^{2}+\sigma_{\mathrm{G} \times \mathrm{E}}^{2}+\sigma_{\text {error }}^{2}}$,

except for the clone variance, which is included in the denominator. From this it follows that $H_{\mathrm{p} 1}^{2}$ must always be larger than $V_{\mathrm{G} \times \mathrm{E}}$, whenever $\sigma_{\text {clone }}^{2}>0$. The crossenvironment correlation CEC,
$\mathrm{CEC}=\frac{\mathrm{COV}}{\sigma_{\mathrm{High}} \times \sigma_{\text {Low }}}$,

appears to be of very different nature, since the product of the standard deviations of the trait within the high and low food environments results in a very different denominator, which does not correspond to the sum of variances used as denominator to calculate $H_{\mathrm{p} 1}^{2}$ and $V_{\mathrm{G} \times \mathrm{E}}$.

\section{Results}

\section{Heritability of plasticity}

Broad- and narrow-sense heritability of plasticity were calculated for the original and for the standardized data. Age at maturity contained no additive variance at all, but the total genetic variance $\left(H_{\mathrm{p} 1}^{2}=\right.$ broad-sense heritability $)$ was significant $\left(H_{\mathrm{p} 1}^{2}=36\right.$ per cent, $P=0.002$, standardized data: $\left(H_{\mathrm{p} 1}^{2}=35\right.$ per cent, $P=0.002$ ).

For adult length, the broad-sense heritability, $H_{\mathrm{p} 1}^{2}$, of plasticity increased with age of the females, while
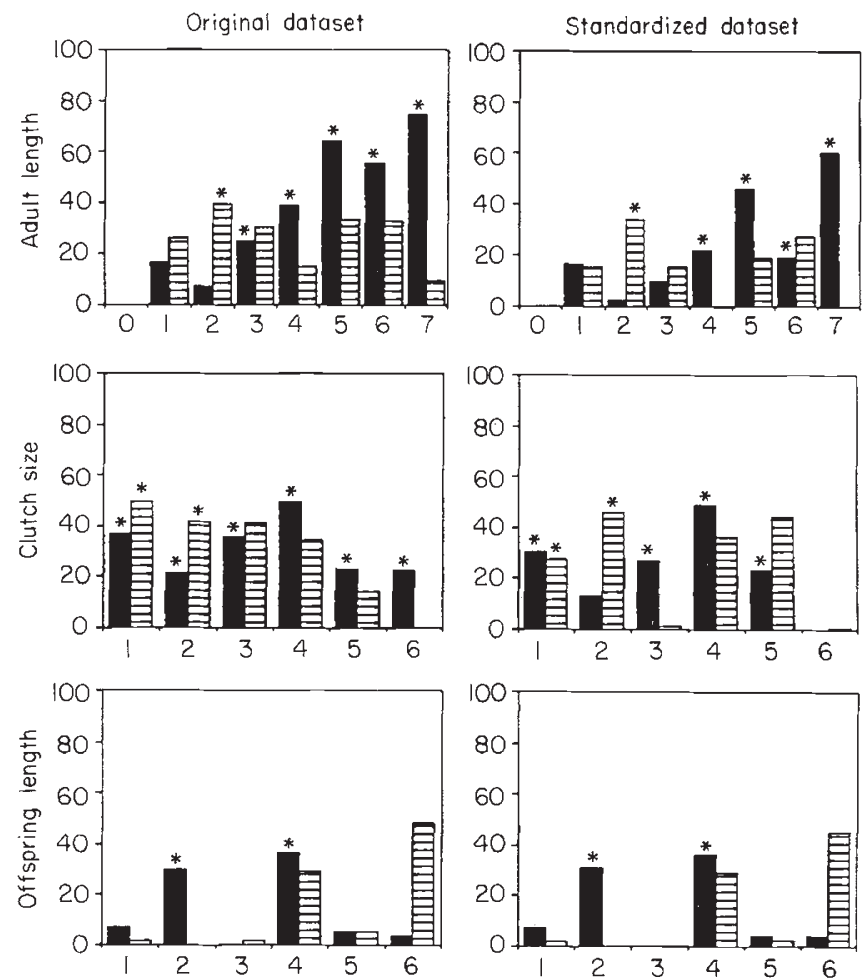

Fig. 1 Heritability of plasticity (per cent) for three groups of life-history traits. Top: body-length from adolescent $(0)$ to 7th adult instar. Middle: clutch sizes 1 to 6 . Bottom: offspring length of clutches 1-6. Black bars: broad-sense heritability, hatched bars: narrow-sense heritability. The three charts on the left: original dataset; right charts: standardized dataset. $* P<0.05$. 
narrow-sense heritability $\left(h_{\mathrm{pl}}^{2}\right)$ did not show any trend (Fig. 1). Heritabilities dropped when the data were standardized, because of the combination of higher heritabilities with higher (four times higher) total variance in high food for these traits. However, the general trend stayed the same after standardization.

The variance components for plasticity of clutch size were fairly constant over the first six clutches (Fig. 1). $h_{\mathrm{p} 1}^{2}$ was smaller than $H_{\mathrm{p} 1}^{2}$ in three cases and larger in three cases. This is what one would expect when they are about equal but subject to random variation. $h_{\mathrm{pl}}^{2}$ decreased with increasing clutch number, a trend which disappeared in the standardized data. This effect could be attributed to the seven times higher total variance in high food. The similar values of $H_{\mathrm{p} 1}^{2}$ and $h_{\mathrm{p} 1}^{2}$, but inconsistent significant levels, illustrate the relatively low power of our design for testing for additive genetic variance (cf. Ebert et al., 1993).

For plasticity of offspring length the pattern looks completely different (Fig. 1). Heritabilities of plasticity were in general low for offspring length compared with other traits. Standardized and original data did not differ because the total phenotypic variance did not differ between environments. In contrast to the within environment heritabilities (cf. Ebert et al., 1993), combined probability tests (Sokal \& Rohlf, 1981) were not significant when for both food levels all six $h_{\mathrm{pi}}^{2}$ probabilities were combined (high food: $\chi^{2}=10.23$, d.f. $=12$, ns; low food: $\chi^{2}=11.8$, d.f. $=12$, ns).

\section{Comparison of clonal means}

The plasticities of the 21 traits are well illustrated in plots of clonal means of the two environments against each other (Fig. 2). The positive correlation of these clonal means, found for most traits, indicated that the relative position of a clone mean in one food level does not change drastically in the other food level (Table 1).

Pearson cross-environmental correlations of clonal means (CEC) were significantly positive for all traits but clutch size 4,5 and 6 (Table 1). This may seem surprising because the scattering of clutch sizes 2-6 (Fig. 2) hardly differed from each other. However, correlations cannot be inferred from inspection of scattergrams when the correlation coefficient is between 0.5 and -0.5 (Sokal \& Rohlf, 1981, page 568) unless the sample size is very large.

All cross-environment correlations for the six offspring lengths and the eight adult lengths were significantly positive (Fig. 2, Table 1). For offspring length, the graphs show a constant pattern for all clutches, and hardly any clone lay outside the cluster, whereas this occurred frequently in the adult length and the clutch size plots. In contrast to adult length and clutch size, the clusters of offspring lengths approached the bisector as instar number increased, indicating that in late clutches offspring lengths were about equally expressed in both feeding conditions.

The plot of age at maturity was dominated by one outlying clone. Therefore, the Pearson correlation coefficient was much higher $(0.66)$ than the Spearman coefficient $(0.23)$.

\section{Comparisons of reaction norm heritability estimates}

The estimates from the three methods used to estimate the heritability of plasticity (Table 1) are plotted against each other in Fig. 3. The plot of the $\mathrm{G} \times \mathrm{E}$ variance components $\left(V_{\mathrm{G} \times \mathrm{E}}\right)$ against those of the heritability of plasticity $\left(H_{\mathrm{p} 1}^{2}\right)$ (Fig. 3a) showed a good agreement of
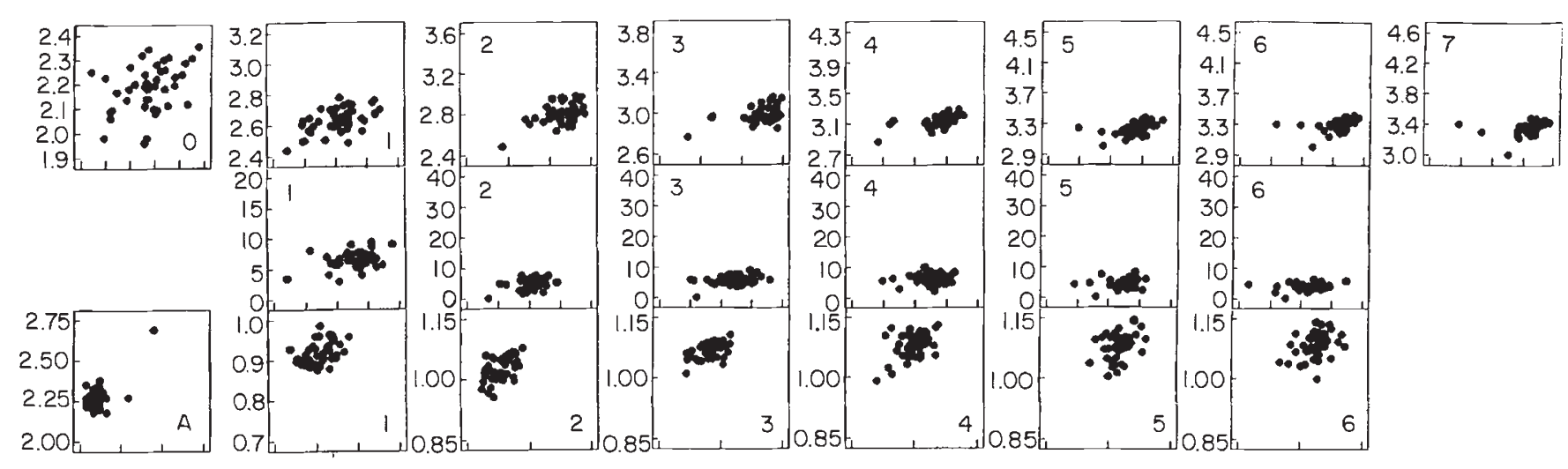

Fig. 2 Plot of low food clonal means (vertical axis) against high food clonal means (horizontal axis) for 21 life-history traits from Daphnia magna. Top row: total body length from adolescent $(0)$ to 7 th adult instar. Middle row: clutch sizes 1-6. Bottom row: age at maturity $(\log$ scale $)(A)$ and offspring lengths of clutches $1-6$. Note that in each graph both axes have the same scaling, but scaling differs between graphs. 
Table 1 Broad-sense heritabilities (in percent) of 21 traits measured in two environments (low and high food), and between environments $\left(\mathrm{G} \times \mathrm{E}\right.$ variance component $=V_{\mathrm{G} \times \mathrm{E}}$, heritability of the cross-environment difference $\left.=H_{\mathrm{p} 1}^{2}\right)$. Last two columns Pearson and Spearman cross-environment genetic correlation coefficients. All 46 clones were included (mother and daughter clones, $n=46)$. Significant values are shown in bold face $(\mathrm{p}<0.05)$. The estimates of heritability in this Table and in Fig. 1 differ somewhat, because broad sense heritabilities in Fig. 1 were calculated only for mother clones

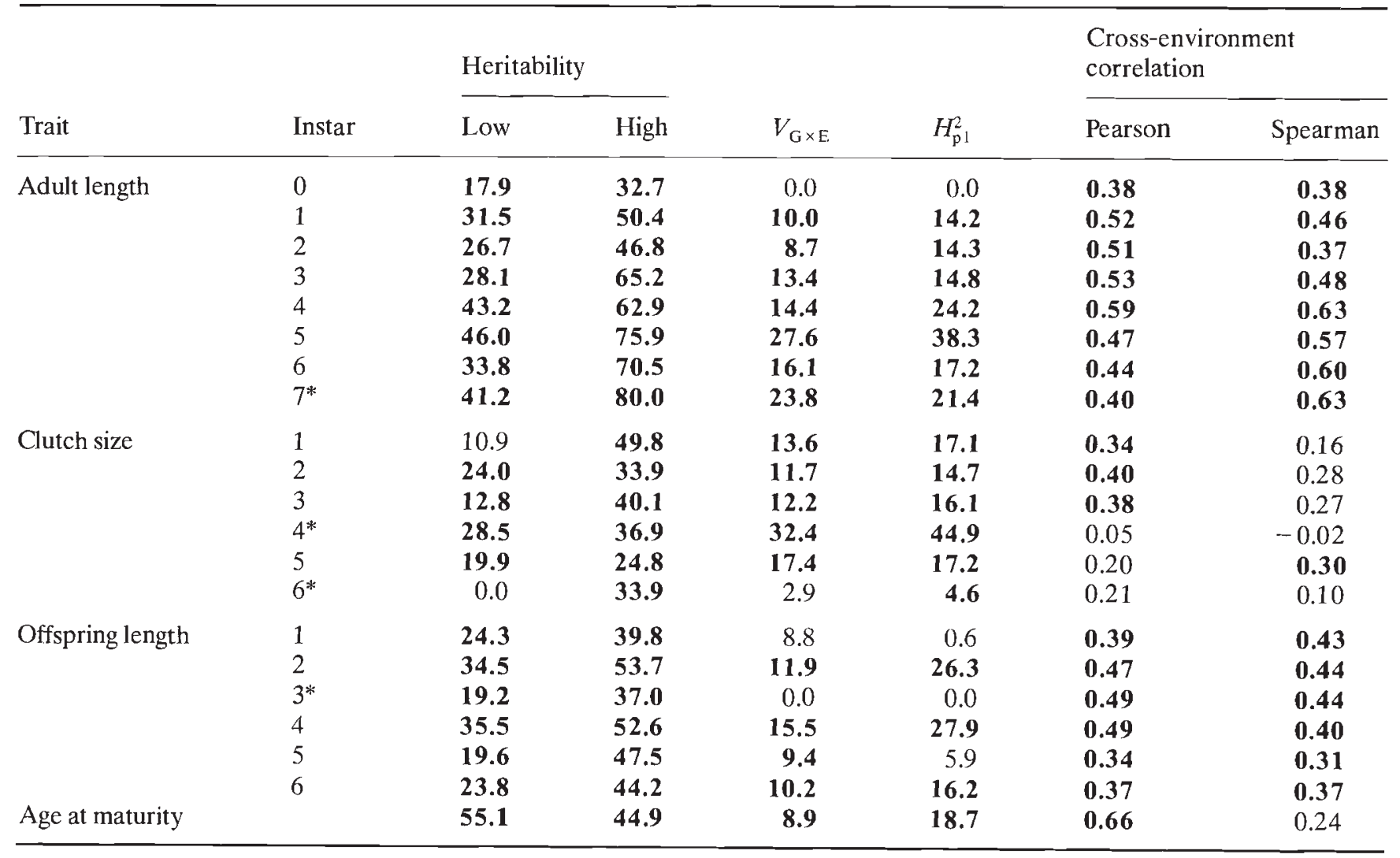

*The reaction norm of these traits are shown in Fig. 5.

both measurements, as it was expected from the statistical interdependences. $H_{\mathrm{pl}}^{2}$ estimates were on average 1.28 times higher than $V_{\mathrm{G} \times \mathrm{E}}$ estimates.

Comparing $H_{\mathrm{p} 1}^{2}$ with the cross-environmental correlation (CEC) indicated that the within trait group (adult lengths, clutch sizes, offspring lengths) variability of estimates is much lower for CEC than for $H_{\mathrm{p} 1}^{2}$, especially for adult length and offspring length (Fig. 3b). The plot of $V_{\mathrm{G} \times \mathrm{E}}$ against CEC (Fig. 3c) is similar to the previous one, but the trait groups cluster more than in Fig. $3 \mathrm{~b}$. The relative stability of the CEC estimates within trait groups allowed separating of trait groups from each other to some extent (Figs 3b, c). A possible reason for the higher stability of CEC estimates across instars for adult and offspring lengths is that successive adult lengths depend strongly on each other and influence the length of offspring (Ebert, 1991), whereas clutch size reflects the current microclimatic conditions more strongly.
No correlations between estimates were found in Fig. 3(b) and (c) as they were in $3(a)$. The only trend within one of the trait groups was the increase of adult length $H_{\mathrm{p} 1}^{2}$ with instar number, depicted in Fig. 1.

The estimates of age at maturity were in Fig. 3(b) and $(\mathrm{c})$ outside the range of the other estimates, which is mainly due to the overestimated CEC (cf. Fig. 2). Due to the large measurement error for time periods (cultures were checked once per day and the estimation errors of birth and maturation time combine), we believe that ages at maturity were poorly estimated and do not draw conclusions from it.

\section{Discussion}

Heritability of plasticity for adult lengths, clutch sizes and offspring lengths differed markedly within and between groups of traits measured over successive 

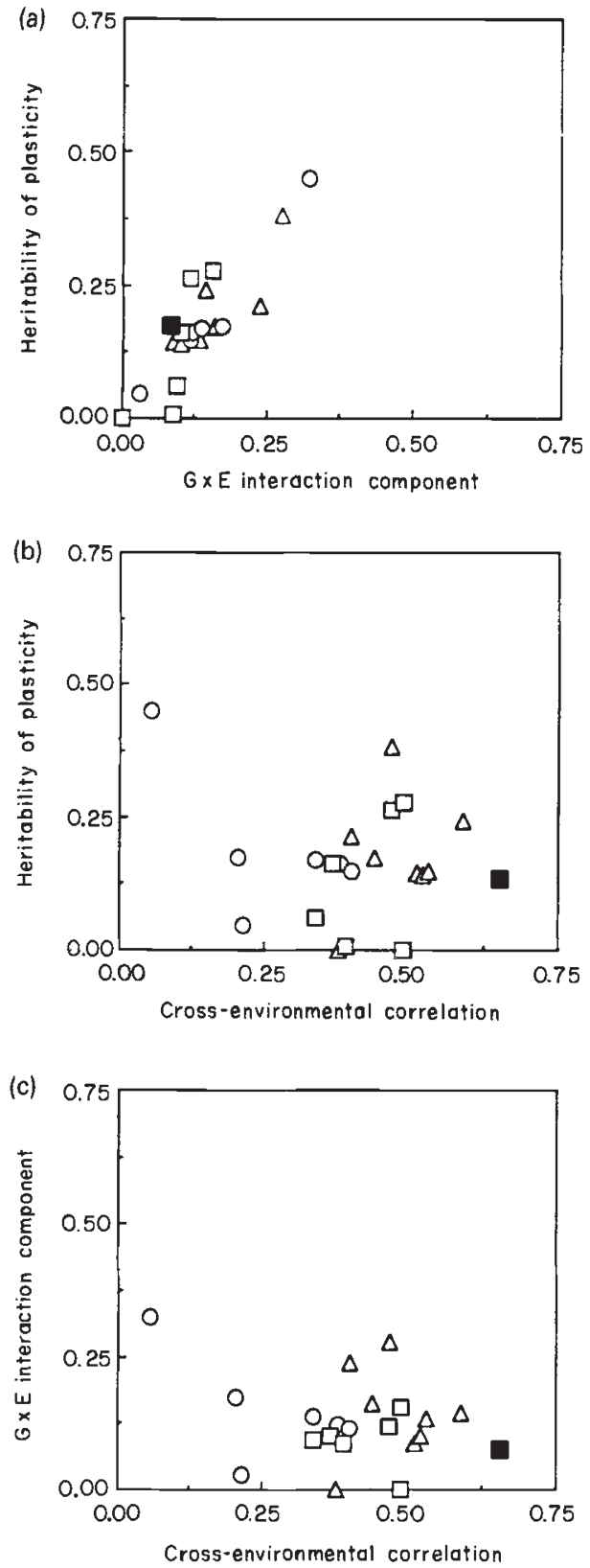

Fig. 3 Estimates of the heritability of plasticity $\left(H_{\mathrm{p} 1}^{2}\right)$, genotype by environment interaction variance component $\left(V_{\mathrm{G} \times \mathrm{E}}\right)$ and cross environment genetic correlation (CEC) of 21 life history traits plotted against each other. (a) $H_{\mathrm{p} 1}^{2}$ against $V_{\mathrm{G} \times \mathrm{E}}$; (b) $H_{\mathrm{p} 1}^{2}$ against $\mathrm{CEC}$; (c) $V_{\mathrm{G} \times \mathrm{E}}$ against CEC. Symbols: triangles: adult lengths; circles: clutch sizes; squares: offspring lengths; black square: age at maturity.

instars. Adult lengths and clutch sizes clearly showed genetic variance for reaction norms, while offspring length showed hardly any genetic variation, although narrow- and broad-sense heritabilities within the two environments were around 30 per cent for offspring lengths (Table 1). Heritability estimates of plasticity appeared to have values between those estimates obtained within the two environments. This was particularly clear for heritabilities of adult length, which increased from 30 to 80 per cent in the high food environment but stayed around 30 per cent in low food.

A plot of the average heritabilities from the high and low food environment against the heritabilities of plasticity revealed a positive trend within each of the three trait groups (Fig. 4), but with clear separation of the three groups. The fact that most points lie above the bisector indicates the low power of estimating heritabilities of plasticity, possibly because developmental noise and measurement error in two environments were combined. This trend was also found for heritability estimates given for morphological traits in Drosophila (Table 2 in Scheiner et al., 1991).

Except for some clutch sizes, narrow-sense heritability was not significant. As already mentioned for the within environment heritability estimates (part 1 of this study; Ebert et al., 1993), for most traits (except adult length 4 to 8) narrow and broad sense heritabilities had similar estimates. For a cyclic parthenogen like Daphnia magna, both heritabilities are important, because with respect to selection, broad-sense heritability is important during asexual reproduction and narrow-sense heritability during sexual phases (Lynch, 1984).

\section{Comparing estimates of phenotypic plasticity}

Although the three methods used to quantify genetic variation of reaction norms are statistically interdependent, some general relations can be seen when single components are considered. High non-genetic variance

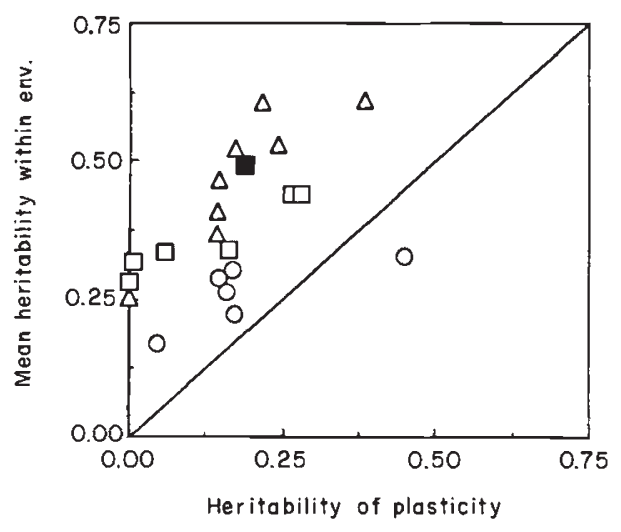

Fig. 4 Plot of the average of the low and the high food broad-sense heritability estimates against the broad-sense heritability of plasticity. For symbols see Fig. 3 . 
(e.g. developmental noise, measurement error) shifts all three estimates toward zero. Genotype by environment interactions do not change CEC, but increase the estimates of $H_{\mathrm{p} 1}^{2}$ and $V_{\mathrm{G} \times \mathrm{E}}$. Genetic covariance does not contribute to $H_{\mathrm{p} 1}^{2}$, but decreases the estimates of $V_{\mathrm{G} \times \mathrm{E}}$ and increases the estimates of CEC. From equation 2 it follows that the $\mathrm{G} \times \mathrm{E}$ component and the genetic covariance are negatively related to each other when the sum of the genetic variances within the two environments is constant. From these relationships it is possible to gain some better understanding of the results, e.g. if $H_{\mathrm{p} 1}^{2}$ and CEC are both low, their estimates are dominated by nongenetic variance. Low $H_{\mathrm{p} 1}^{2}$ and high CEC estimates indicate high total genetic variance, but only a little of it is due to genotype by environment interactions, while high $H_{\mathrm{p} 1}^{2}$ and low CEC indicate high genetic variation for plasticity. Therefore one cannot expect a correlation between $H_{\mathrm{p} 1}^{2}$ and CEC estimates (Fig. 3b). The relationship between $H_{\mathrm{p} 1}^{2}$ and $V_{\mathrm{G} \times \mathrm{E}}$ is rather simple and suggests a positive correlation between both estimates, as it was found (Fig. 3a). However, in the real world, variance component estimates tend to have large errors (Falconer, 1981). Since we estimated the variance components in three different ways, one-way ANOVA for $H_{\mathrm{p} 1}^{2}$, two-way ANOVA for $V_{\mathrm{G} \times \mathrm{E}}$, and variance covariance matrix for CEC, the estimation error of each method combines when estimates are compared, e.g. although $H_{\mathrm{p} 1}^{2}$ should be larger than $V_{\mathrm{G} \times \mathrm{E}}$ whenever the clone variance component is larger than zero, in some cases $V_{\mathrm{G} \times \mathrm{E}}$ was equal to or larger than $H_{\mathrm{p} 1}^{2}$ (e.g. adult length 7 , clutch size 5 and offspring length 1 and 5, Table 1). Comparing directly the variance components obtained by different methods of our 21 traits, with the relations given in equations 1 and 2, we found large deviations in both directions, explaining the discrepancy of the $V_{\mathrm{G} \times \mathrm{E}}$ and $H_{\mathrm{p} 1}^{2}$ estimates. We can think of several reasons for these deviations. One source of deviation between the oneway and the two-way ANOVA variance estimates is due to our method to calculate the data set of the crossenvironmental differences. We subtracted each of the three low food replicates from one of the three high food replicates. Thus, the mean and variance of the clonal differences are subject to (unbiased) combination effects, which do not occur in the two-way ANOVA. These combination effects could be reduced by using the mean estimate of repeated calculations with different combinations of low and high food replicates. Another source of error lies in the estimation of the variance components itself. Since our data set was not completely balanced, we did not calculate the variance components directly from the mean squares, but used the maximum likelihood method (SAS, PROC VARCOMP). Further, the quality of variance compon- ent estimates depends very strong on sample size, illustrated for the $H_{p 1}^{2}$ estimates by comparing the standardized data set in Fig. 1 (23 mother clones) with the $H_{\mathrm{p} 1}^{2}$ estimates in Table 1 ( 46 clones). In the most diverging case the heritability dropped from 60 to 21 per cent (adult length 7).

In summary, we prefer the $H_{\mathrm{p} 1}^{2}$ estimate over the other two estimates of quantifying genetic variance of reaction norm, because it is the purest measurement of plasticity itself, i.e. it estimates the variance component of the slope of the reaction norm and does not depend on the mean clonal variance. Although, the three proposed methods to quantify the genetic variation of reaction norms are statistically interdependent from each other, the complexity of these dependencies and the low power of estimating variance components precisely should lead us to caution in concluding from one reaction norm estimate to another. On the other hand, the statistical interdependence of the three methods from each other, allow to calculate all variance component estimates by use of a minimum of information, i.e. the genetic and nongenetic variances in both environments and the genetic covariance.

\section{Crossing reaction norms}

All three methods used here to quantify the genetics of phenotypic plasticity allow some kind of prediction of non-parallel reaction norms, although none of them tests directly for differences in rank order as does the Spearman correlation coefficient. However, a comparison of the Spearman and the Pearson correlation coefficients show a good agreement between the two, with the one exception of age at maturity.

According to Gupta \& Lewontin (1982), the most useful way to analyse reaction norms is to plot them. As an example, four traits with different combinations of plasticity estimates (marked in Table 1) are used as examples (Fig. 5). The differences in CECs (c4 $<\mathrm{c} 6<\mathrm{a} 7<\mathrm{o} 3$; Fig. 4) could not be seen easily. The differences in $H_{\mathrm{p} 1}^{2}$ for clutch size $4\left(H_{\mathrm{p} 1}^{2}=0.45\right)$ and clutch size $6\left(H_{\mathrm{p} 1}^{2}=0.05\right)$ were not reflected in these graphs. Clearly, crossing reaction norms are common in all four graphs, but it is hardly possible to analyse these reaction norm bundles visually.

\section{Conclusion}

The genotype-environment-interaction component and the heritability of the cross-environment trait difference gave corresponding results, while the crossenvironment genetic correlation gave a qualitatively different estimate of plasticity. The heritability of the difference in the expression of one trait in two environ- 

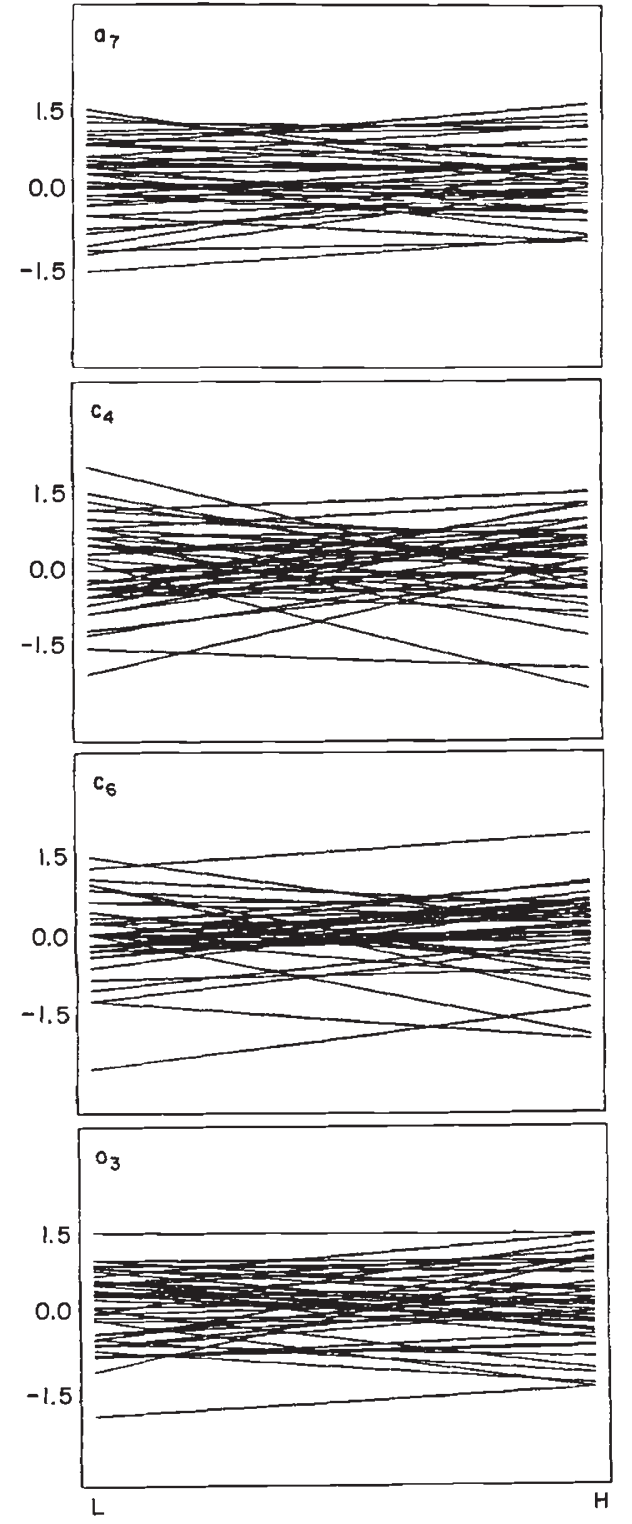

Fig. 5 Reaction norms for adult length instar 7 (a7), clutch size 4 (c4) and 6 (c6) and offspring length instar $3(\mathrm{o} 3)$. Standardized data are used for graphs (mean $=0$, variance $=1$ ). Each line represents the reaction norm of one clone between low $(\mathrm{L})$ and high $(\mathrm{H})$ food environments. The four traits are marked $\left(^{*}\right)$ in Table 1.

ments (our $H_{\mathrm{p} 1}^{2}$ ) comes closest to our intuitive idea of heritable variation in how genotypes would respond to different selection regimes in two environments. It corresponds to the idea that the slope of a reaction norm is a heritable trait by itself (Gavrilets, 1986; de Jong, 1990). Future research on the genetics of phenotypic plasticity needs to focus on the evolutionary interpretations of different methods to quantify genetic variation in reaction norms, because the prediction of the response to selection is the factor we are interested in.

\section{Acknowledgements}

We thank S. C. Stearns, P. D. N. Hebert, A. L. Rypstra and two anonymous reviewers for comments on various earlier versions of the manuscript and for improving the language. F.H.C. Marriott helped us with the statistical analysis. Special thanks to M. Bürki for laboratory assistance. The work was supported by Swiss Nationalfond grant No.3.643.0.87 and No.3100028511-01.

\section{References}

APPLETON, R. D. AND PALMER, A, R. 1988. Water-borne stimuli released by predatory crabs and damaged prey induce more predator-resistant shell in a marine gastropod. Proc. Natl. Acad. Sci., 85, 4387-4391.

BECKER, W. A. 1964. Heritability of a response to an environmental change in chickens. Genetics, 50, 783-788.

DE JONG, G. 1990. Quantitative genetics of reaction norms. J. Evol. Biol., 3, 447-468.

DoDson, S. 1989. Predator-induced reaction norms. Bioscience, 39, 447-452.

EBERT, D. 1991. The effect of size at birth, maturation threshold and genetic differences on the life-history of Daphnia magna. Oecologia (Berl.), 86, 243-250.

EBERT, D., YAMPOLSKY, L. AND STEARNS, S. C. 1993. Genetics of life-history in Daphnia magna: 1. Heritabilities at two food levels. Heredity, 70, 335-343.

FALCONER, D. S. 1981. Introduction to Quantitative Genetics, 2nd edn. Longman, London.

GAVRILETS, s. 1986. An approach to modelling the evolution of populations with consideration of genotype-environment interaction. (Trans. from Russian) Soviet Genetics, 22, 28-36.

GEBHARDT, M. D. AND STEARNS, S. C. 1988. Reaction norms for developmental time and weight at eclosion in Drosophila mercatorum. J. Evol. Biol., 1, 335-354.

GUPTA, A. P. AND LEWONTIN, R. C. 1982. A study of reaction norms in natural populations of Drosophila pseudoobscura. Evolution, 36, 934-948.

LYNCH, M. 1984. The limits to life history evolution in Daphnia. Evolution, 38, 465-482.

VAN NOORDWIJK, A. J. 1989. Reaction norms in genetical ecology. Bioscience, 39, 453-458.

ROBERTSON, F. W. 1960. The ecological genetics of growth in Drosophila 2. Selection for large body size on different diets. Genet. Res., 1, 305-318.

SAS INSTITUTE INC. 1990. SAS/STAT, Version 6, Cary, NC: SAS Institute Inc.

SCHEINER, S. M., CAPLAN, R. L. AND LYMAN, R. 1991. The genetics of phenotypic plasticity III. Genetic correlations and fluctuating asymmetries. J. Evol. Biol., 4, 51-68. 
SCHEINER, S. M. AND GOODNIGHT, C. J. 1984. The comparison of phenotypic plasticity and genetic variation in populations of the grass Danthonia spicata. Evolution, 38, 845-855.

SCHEINER, S. M. AND LYMAN, R. 1989. The genetics of phenotypic plasticity I. Heritability. J. Evol. Biol., 2, 95-107.

SOKAL, R. R. AND ROHLF, F. J. 1981. Biometry, 2nd edn. Freemann, New York.

STEARNS, S. C. 1989. The evolutionary significance of phenotypic plasticity. Bioscience, 39, 436-445.

VIA, s. 1984. The quantitative genetics of polyphagy in an insect herbivore. I. Genotype-environment interaction in larval performance on different host plant species. Evolution, 38, 881-895.

VIA, S. 1987. Genetic constraints on the evolution of phenotypic plasticity. In: V. Loeschke (ed.) Genetic Constraints on Adaptive Evolution, Springer, Berlin, Heidelberg, pp. 47-71.

WOLTERECK, R. 1909. Weitere experimentelle Untersuchungen über Artveränderung, speziell über das Wesen quantitativer Artunterschiede bei Daphniden. Verhandl. $d$. Deutsch. Zool. Gesellschaft, 1909, 110-173. 